Dhaka Univ. J. Sci. 60(2): 271-275, 2012 (July)

\title{
Origin of the Larger Values of Total Cross Section for Helium Scattering from Isolated Atomic Steps
}

\author{
K. S. Sultana ${ }^{1}$, W Allison ${ }^{2}$ and M. Z. Hafiz ${ }^{3}$ \\ ${ }^{1}$ Department of Physics, University of Chittagong, Chittagong-4331, Bangladesh \\ ${ }^{2}$ Cavendish Laboratory, University of Cambridge, JJ Thomson Avenue, Cambridge CB3 OHE, UK \\ ${ }^{3}$ Institute of Information Technology, Dhaka University, Dhaka-1000, Bangladesh
}

Received on 10. 02. 2012. Accepted for Publication on 12. 03. 2012.

\begin{abstract}
The effect of surface relaxation and the electronic re-arrangement in the vicinity of a step on the total step cross section for helium scattering is investigated. A realistic helium interaction potential at a $\mathrm{Cu}(001)$ step is modeled by summing non-spherical pair potentials which allows for the possibility of varying the smoothing across the surface due to the itinerant aspects of the surface electronic structure. Numerical calculations reveal a significant increase in the magnitude of the total step cross section with large charge re-arrangement in the vicinity of the step. Also, the relaxation of surface layers has no effect whatsoever. The present study clearly shows that the origin of the experimentally observed large step cross section is the hard wall scattering from charge re-arrangement in the proximity of the step. Further, the charge re-arrangements probed by thermal helium atoms must be greater than predicted by pairwise models.
\end{abstract}

\section{Introduction}

A detailed understanding of surface structure is an important prerequisite for explaining many technological processes, such as thin film growth, crystal growth, molecular self assembly and heterogeneous catalysis. The properties of thin metal or semiconductor films on solid substrates are of major theoretical, experimental and technological interest. The structure of these films influences their physical and chemical properties. In this respect, helium atom scattering is a particularly useful method to investigate structural and dynamical properties of a surface. It is non destructive and has negligible penetration depth. Therefore, helium atoms probe only the outer layer. In addition to being sensitive to local surface features, it resolves the global surface structure. Accordingly, helium atom diffraction method provides a better average of the overall topography than direct imaging techniques, such as scanning tunneling microscopy (STM). Moreover, diffraction readily allows for monitoring adlayer growth in situ and at various temperatures and therefore can easily follow its temporal evolution.

In fact the analysis of helium scattering cross sections significantly advanced our understanding of the surface corrugation [1-3], distribution and dynamics of defects and adsorbates on the surface [4-6], surface diffusion [7] and island formation [8]. The very large scattering cross sections [9] of adsorbed gases (of the order of 100A) make it possible to study the structure of adsorbate covered surfaces, migration of low-coverage adsorbates on surfaces. Similarly, large cross sections (of the order of $12 \AA$ ) $[10,11]$ for diffuse helium scattering from steps on metal surfaces enables us to characterize randomly stepped surfaces and to study the epitaxial growth processes [12]. Experimental studies have shown that the magnitude of step edge cross section for different metal surfaces are rather large compared to the atomic dimension of the crystal face [10,11]. It contradicts the notion that the total step cross section should be equal to the atomic dimension since total cross section for helium scattering from stepped surfaces are defined in terms of an area per unit length.

We have carried out an investigation to understand the origin of the larger values of total step cross section observed experimentally. Our previous studies $[13,14]$ have focused mainly on the energy dependence of total cross sections for scattering of helium atoms from isolated atomic steps on metal surfaces. We found that the repulsive part of the potential dominates the scattering mechanism and that there is no effect from the attractive part of the potential in the larger values of total cross section in step scattering. Moreover, total cross section increases with energy of the helium atoms. It is generally believed that the step potential arises from the smear out of the surface charge around the step edge $[15,16]$. Therefore, electronic re-arrangement in the vicinity of the step may play a key role in the measurement of total cross section for helium scattering. In this study we have explored the two possible factors: (a) relaxation of the atoms in the surface layer and (b) electronic re-arrangement at the step which can play a role in causing the above mentioned effects. 
Here we present the calculated values of step scattering cross sections for $\mathrm{Cu}(001)$ surfaces making use of realistic potentials that reproduce the scattering from low index planes and are created from non-spherical, pairwise terms. The smoothing effect of the metallic surface electrons is presented by a single, site dependent anisotropy parameter $\gamma$. The results clearly show that the origin of the large step cross section is the hard wall scattering from charge rearrangement in the proximity of the step. Further, the magnitude of the total cross section is not affected by surface relaxation at all.

\section{Method}

To calculate the total step cross section we need to simulate helium scattering with a realistic helium surface interaction potential for the step region.

The atom surface interaction is dominated by single scattering events in helium atom scattering, except at low energies and/or near grazing incidence. Diffraction experiments can explore both the lateral and vertical morphology either by varying the in-phase wave vector, $\boldsymbol{k}_{\|}$, giving a spot profile analysis, or by varying the perpendicular wave vector $\boldsymbol{k}_{z}$ giving a lattice rod scan. This paper concentrates on the latter. Lattice rod scans may be performed either with a fixed beam energy, by varying the scattering geometry, or by varying the beam energy with a fixed scattering geometry [17]. The principles are the same for both types of experiments. The specular lattice rod scans are used in the measurements of helium step cross sections.

The total cross sections for step scattering is given in terms of a step width $D$ along the step edge contour. The expression for the in-phase fractional specularly reflected helium intensity [13]

$$
I / I_{0}=(1-D S)^{2}
$$

is used to obtain $D$. Here $S$ is the step density, $I_{0}$ is the specular intensity from a clean surface (i.e., $S \approx 0$ ) and $I$ is the specular intensity from a stepped surface with step density $S$. If $S$ is defined as the total step edge length per unit area than a fraction $D S$ of the surface will scatter diffusely.

The specular lattice rod scan i.e., the oscillatory behaviour of helium specular intensity or peak height as a function of perpendicular momentum change $\left(\Delta \boldsymbol{k}_{z}\right)$ enables a rough estimate of the step density and the terrace width distribution. The ratio $I / I_{0}$ is obtained by measuring the inphase specular peak height for a surface with steps and with a defect free surface. Thus one can experimentally determine $S$ and $I / I_{0}$ and then directly obtain $D$ using equation (1).

The procedure to calculate the total step cross sections for helium scattering from stepped surfaces with a known step density is analogous to the experimental one. The method has been described in details elsewhere $[13,14]$. A realistic helium surface interaction potential model $[14,18]$ easily applicable to complex systems, such as stepped surfaces, using additive, non-spherical, pairwise potentials with a few fixed parameters and only one free parameter has been used to calculate helium step interaction potential on $\mathrm{Cu}(001)$ surfaces. The model has the scope to include surface relaxation if the displacements of the surface atomic planes are known. Further, different smoothing at different atomic sites is possible to simulate the effect of electronic re-arrangements at step sites.

In order to calculate the cross section we use a two layer regularly stepped $\mathrm{Cu}(001)$ surface. The step density of the surface can be varied by changing the terrace length. The upper terrace length is taken to be equal to the lower terrace length for simplicity. The simulations of helium scattering from the above mentioned surface for calculating specular intensity $(I)$ and from pristine $\mathrm{Cu}(001)$ surface for calculating $\left(I_{0}\right)$ have been performed using modified sudden approximation [19]. The procedure has been described elsewhere [14,19].

\section{Results and Discussion}

In our previous study [13] we have used helium-step interaction potential using the same smoothing factor $\gamma=1.5$ for all atoms as for the atoms on a flat $\mathrm{Cu}(001)$ surface. The magnitude of the total cross section for the above potential using the procedure described in the previous section is significantly small (around 5 to $8 \mathrm{~A}$ ) than the experimental value (around $12 \AA$ ). In order to explain why the observed total step cross section is larger than that of the calculated value one needs to look for physical effects not included in the model so far. Accordingly the results presented here show the effect of surface relaxation and electronic re-arrangement at step site on the total step scattering.

To incorporate surface relaxation in the helium-step interaction potential it is necessary to know the positions of the atoms on a relaxed $\mathrm{Cu}(001)$ stepped surface. However, no experimental work has been performed to provide an exact guide to the positions of the atoms on a relaxed surface layer of a $\mathrm{Cu}(001)$ stepped surface, in particular at the topmost layer. Surface relaxation data measured by LEED for a pristine surface is available [20] for $\mathrm{Cu}(001)$. This gives a contraction between the first and second layer (i.e., $d_{12}$ ), expansion between the second and third layer (i.e., $d_{23}$ ), expansion between the third and fourth layer (i.e., $d_{34}$ ) and then normal bulk spacing. It is an oscillatory relaxation of interlayer spacings as expected for an open metal surface [21]. In the relaxed $\mathrm{Cu}(001)$ surface the atoms near the steps edge will not be situated at the expected bulk lattice positions. So we interpolate the positions of the atoms near the step edge using the relaxed interlayer spacings. The relaxation of the atoms near the step will be confined within a finite distance $l$ as shown in fig. 1 which schematically illustrates the seven top layers of an isolated step on a $\mathrm{Cu}(001)$ relaxed surface. A smooth function between two adjacent levels within a range $l$ will be adequate to represent the relaxation adjacent to the step 
edge. We use a simple cosine curve as an interpolation function. The range $l$ is used as a variable parameter, and we define $l / 2$ as the "relaxation width". Fig. 2 shows the variation of step cross section with relaxation width $l / 2$ at a particular beam energy $\left(k_{\mathrm{i}}=6.97 \AA^{-1}\right)$ for normal incidence.

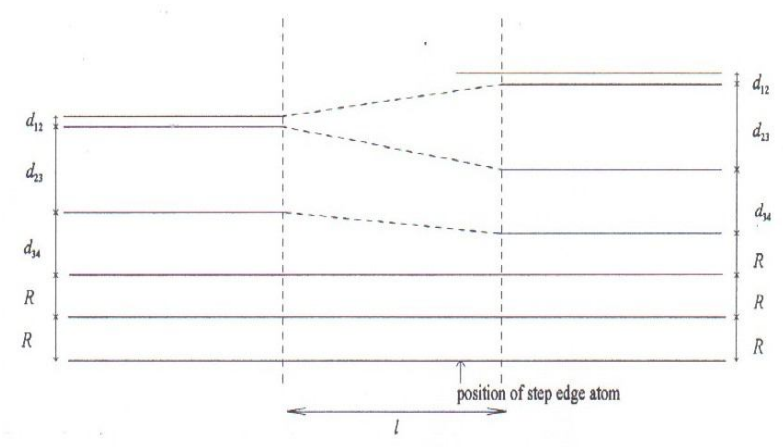

Fig 1. A schematic diagram showing the seven upper most layers of an isolated up step of $\mathrm{Cu}(001)$. The first two layers are contracted, the second and third layers expanded, third and fourth layers are also expanded but $d_{34}<d_{23}$ (inter layer spacings) and starting from the fifth layer the layers are in their normal bulk positions with regular spacing $R=1.803 \AA$.

It is evident that there is no significant change of step cross section even for relaxation of the atoms up to four atoms away from the step edge on either side. An expanded scale has been used to show the variation of cross section with $l / 2$ in fig. 2 .

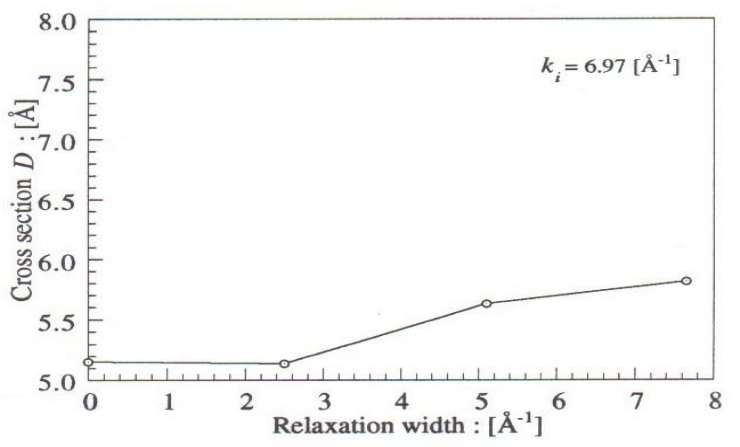

Fig. 2. Variation of cross section with relaxation width $l / 2$ for a particular beam energy corresponding to the incident helium wavevector $k_{\mathrm{i}}=6.97 \AA^{-1}$.

We explore the effect of electronic re-arrangement at the topmost layer of the surface. In our potential model, the electronic re-arrangement of the topmost layer metal atoms is described by the smoothing parameter $\gamma$. Until now we have used $\gamma=1.5$ for all the atoms. But $\gamma$ for step edge atoms may have different values than the terrace atoms. So we varied the smoothing parameter of the step atom from $\gamma_{\mathrm{s}}=0.0$ to $\gamma_{\mathrm{s}}=5.0$ keeping $\gamma=1.5$ for all other atoms. Figs. 3 and 4 show helium-step interaction potential for four different smoothing parameters: $\gamma_{\mathrm{s}}=0.0,2.0,3.0$ and 5.0 for the step edge atom. The step edge atom is positioned at $x=$ $0 \AA$ on the one dimensional step for which the interaction potential is calculated. The width of the step is taken to be $22.775 \AA$. The figures show that increasing the value of the smoothing parameter of the step edge atom changes the helium-step interaction potential significantly. Step cross sections for different beam energies using helium-step interaction potentials for different values of $\gamma_{\mathrm{s}}$ for the step edge atom have been calculated. Fig. 5 shows the variation of step cross section with beam energy for the various cases. We obtain a significant increase in the magnitude of the cross section with increasing $\gamma_{s}$. Fig 6 shows the variation of total cross section as a function of $\gamma_{\mathrm{s}}$ for step edge atom at a particular beam energy $\left(k_{i}=6.97 \AA^{-1}\right)$. Interestingly, the magnitude of $D$ reaches the typical values of $12 \AA$ observed in the experiment for values of $\gamma_{\mathrm{s}}$ between 4 and $5 \AA$. The larger value of $D$ for bigger $\gamma_{\mathrm{s}}$ arises because the hard wall potential extends over a much wider range than in the earlier calculations [13]. However, the potentials for $\gamma_{\mathrm{s}}=3$ and for $\gamma_{\mathrm{s}}$ $=5$ in fig. 3 are somewhat unrealistic. A bigger value of $\gamma_{\mathrm{s}}$ introduces corrugation in the potential near the step edge. However, there is no experimental data available to confirm this. Even if this is not the case (i.e., there is no significant corrugation at the vicinity of the step) then different values of $\gamma$ for different atoms across the step will reduce the corrugation (as found in figs. 3 and 4). Also, $\gamma$ for the topmost layer atoms adjacent to the step edge atom may well have different values than the $\gamma$ from the atoms in other layers due to their different geometrical co-ordination and environment.

\section{Conclusion}

Earlier study [13] confirmed that the step scattering is dominated by the hard wall of the scattering potential. There is little effect due to the attractive part of the scattering potential. If there is any effect of the attractive potential in total step cross section then it is secondary. These considerations and the present results lead us to conclude that the large experimental value of $D$ is due to the very large charge rearrangement in the vicinity of the step. In fact a bigger value of $\gamma_{\mathrm{s}}$ for step edge atom in our calculation provides a larger value of $D$, of the same order as the experimentally observed value. Our study shows clearly that the origin of the large step cross section is the hard wall scattering from charge rearrangement in the proximity of the step. Further, the charge re-arrangement probed by thermal helium atoms must be greatest than expected and greater than predicted by pairwise models. 

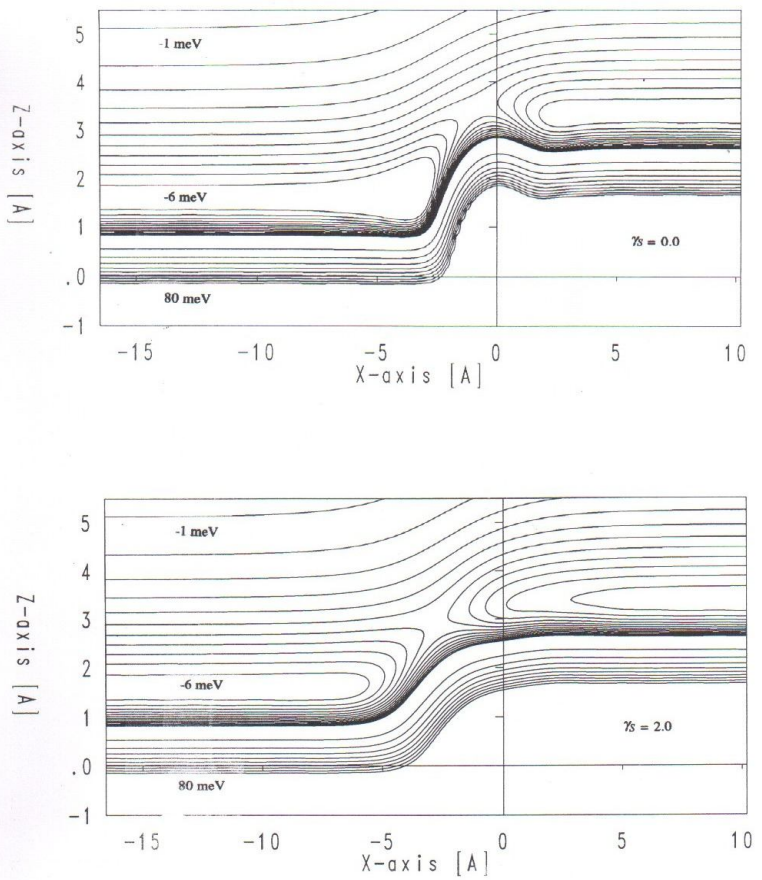

Fig. 3. Contour plots of potentials for an up step of $\mathrm{Cu}(001)$. The $\mathrm{z}$-axis is perpendicular to, and the $\mathrm{x}$-axis parallel to, the surface. The smoothing factor for the step edge atom in the upper plot is $\gamma_{\mathrm{s}}=0.0$ and for the lower plot, $\gamma_{\mathrm{s}}=2.0$. For the rest of the atoms a value of $\gamma=1.5$ has been used in both cases.
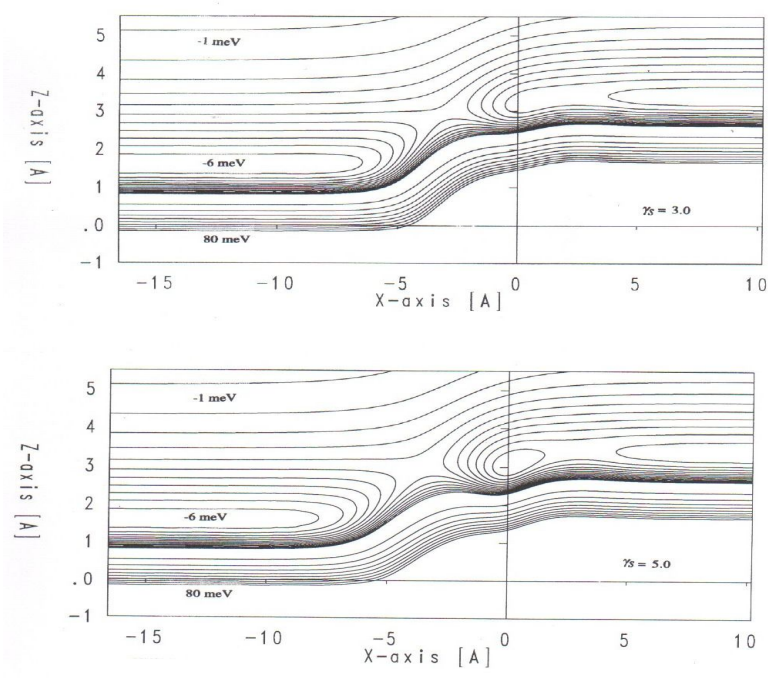

Fig. 4. Contour plots of potentials for an up step of $\mathrm{Cu}(001)$. The $\mathrm{z}$-axis is perpendicular to, and the $\mathrm{x}$-axis parallel to, the surface. The smoothing factor for the step edge atom in the upper plot is $\gamma_{\mathrm{s}}=3.0$ and for the lower plot, $\gamma_{\mathrm{s}}=5.0$. For the rest of the atoms a value of $\gamma=1.5$ has been used in both instances. Observe that the larger values of $\gamma_{\mathrm{s}}$ give rise to corrugation in the vicinity of the step edge.

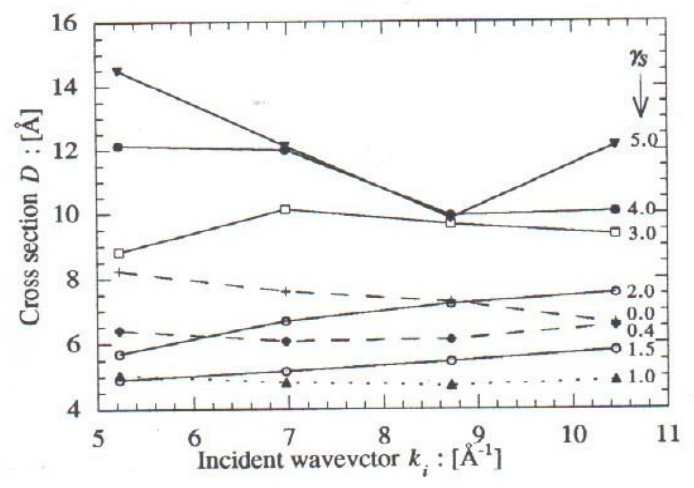

Fig. 5. Variation of step cross section with incident wavevector, or equivalently helium beam energy, for different values of smoothing parameter $\left(\gamma_{\mathrm{s}}\right)$ for step edge atom.

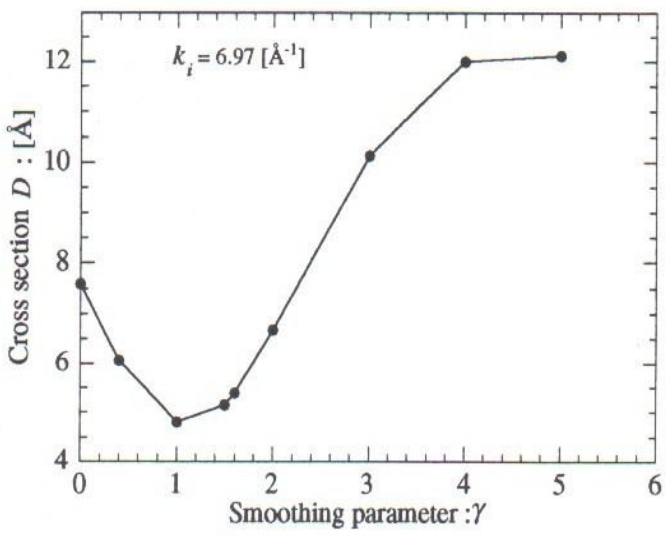

Fig. 6. Variation of step cross section with smoothing parameter for a particular beam energy corresponding to $k_{i}=6.97 \AA^{-1}$.

Careful experiments are required to prove our prediction that the step cross section increases with energy. Also, new calculations can be carried out using a step potential with different $\gamma$ for different atoms on the top most layer of the isolated step and investigate the step cross section in more detail. Such a study is possible using our method of calculating realistic helium-step interaction potential created from non-spherical pairwise terms with the scope of a site dependent anisotropy parameters $\gamma$ to represent the metallic smoothing.

1. Reider K. -H., Helium Atom Scattering from Surfaces, Springer Series in Surface Sciences, Vol. 27, Springer-Verlag, Berlin, pp. 41-71, 1992.

Hedgeland H., P. R. Kole, H. R. Davies, A. P. Jardine, G. Alexander, W. Allison and J. Ellis, Phys. Rev. B 80, 12426, 2009.

2. Choi B. H., A. P. Graham, K. T. Tang and J. P. Toennies, J. Chem. Phys. 112(23), 10538, 2000. 
3. Riely D. J., A. P. Jardine, G. Alexandrowicz, H. Hedgeland, J. Ellis and W. Allison, J. Chem. Phys. 128, 154712, 2008.

4. Becker J. S., R. D. Brown, D. R. Killelea, H. Yuan and S. J. Sibener, PNAS, 108(3), 977, 2011.

5. Freedman, M. A., A. W. Rosenbaum and S. I. Sibener, Phys. Rev. B 75,113410, 2007.

6. Dastoor P., M. Arnott, E. M. McCash and W. Allison, Surf. Sci., 272, 154, 1992.

7. Fratesi G., G. Alexandrowicz, M. I. Triuni, G. P. Brivio and W. Allison, Phys. Rev. B 77, 235444, 2008.

8. Poelsema B. L. K. Verheij and G. Comsa, Phys. Rev. Lett. 49, 1713, 1982.

9. Traeger F., Chem. Phys. Chem. 7, 1006, 2006.

10. Sa'nchez and S. Ferrer, Surf. Sci. 187, L587, 1987.

11. Verheij L. K., B. Poelsema and G. Comsa, Surf. Sci. 162, $585,1985$.

12. Yinnon A. T., D. A. Lidar, I. Farbman, R. B. Gerber and G. Comsa, J. Chem. Phys. 106(10), 4228, 1997.
13. Sultana K. S. and M. Z. Hafiz, J. Bangladesh Academy of Sci. 34(1), 23, 2010.

14. Sultana K. S., Chittagong J. Sci. 25(1), 95, 2001.

15. Wang X. Y., X. J. Shen and R. M. Osgood, Phys. Rev. B, 56, 7665, 1997.

Stumpf R. and M. Scheffler, Phys. Rev. Lett. 72, 254, 1994.

16. Schiller F., M. Ruiz-Ose‘s, J. Cordon And J. E. Ortega, Phys. Rev. Lett. 95, 066805, 2005.

17. Dastoor P. and W. Allison, Surf. Sci., 433, 99, 1999.

18. Sultana K. S. Ph. D. Thesis, Analysis of Helium Scattering from Stepped and Growing Surfaces, Department of Physics, University of Cambridge, 1997.

19. Hinch B. J., Surf. Sci. 221, 346, 1989.

20. Davis H. L and J. R. Noonan, J. Vac. Sci. Techcol. 20, 842, 1982.

21. Zangwil A., Physics at Surfaces, Cambridge University Press, pp.26, 69, 1988. 